\title{
Quartär und Ingenieurgeologie in der Schweiz
}

\begin{abstract}
:
Engineering geology problems very often concern quaternary sediments or the underlying surface of bedrock formed by glacial erosion. To get enough information for building, road construction, tunnelling etc., a tremendous amount of information has been collected during the last 30 years in Switzerland including detailed geological mapping, boreholes, laboratory tests, and data on groundwater. Why did not our knowledge of the Quaternary get more adavantages from all this activity? Two examples shall demonstrate that engineering geology has priorities very different from those of quaternary stratigraphy. It is therefore extremely difficult to get support for purely scientific research from the assignor of a building contract. However, better cooperation between engineering geology and quaternary sciences would be helpful to everybody concerned.
\end{abstract}

\section{Key words:}

Quaternary - Engineering Geology - Anisotropy of Soils Glacial Compaction of Soils

Geologie kann im Feld oder Labor auf verschiedensten Ebenen betrieben werden. Das Motiv für die Arbeit kann reine wissenschaftliche Neugierde sein und das Ziel im Aufbau theoretischer Modelle bestehen. Die Untersuchungen können umgekehrt aber auch zu wesentlichen Teilen zweckgerichtet sein wie in der Ingenieurgeologie und Hydrogeologie. Hier treffen wir auf meist eng lokalisierte Probleme, welche mit Hilfe von Aufschlüssen und Versuchen sowie mit der Anwendung des theoretischen Wissens gelöst werden sollen. Der Geologe möchte Grundlagen liefern für die vielfältigsten Fragen genannt seien etwa Tunnel- und Strassenbau, Bergbau, Probleme der Hangstabilität, Stauwerke, Wasserversorgung, heute in zunehmendem Maße auch die Suche nach geeigneten Deponieplätzen oder andere Aspekte des Umweltschutzes.

In der Schweiz trifft der Ingenieurgeologe dabei sehr häufig auf Lockergesteinsprobleme und auf die Frage des Verlaufs der Felsoberfläche, er wird sich also mehr als die meisten andern Geologen mit dem Quartär beschäftigen müssen. Wie wirkt sich aber dieser Kontakt aus, und wo liegen die Schwerpunkte?

Bevor wir uns mit der heutigen Situation beschäftigen, soll ein geraffter Rückblick versucht werden.

\section{Verhältnisse bis um 1960}

Bis etwa zum Ersten Weltkrieg wurden in unserem Lande an unzähligen Stellen, aber meist in bescheidenen Kubaturen, Rohstoffe abgebaut, so auch Kies, Sand, Lehm, Schieferkohle, Moräne, Quelltuff usw. Da beim Abbau nur wenige Maschinen zur Verfügung standen, erfolgte er gemächlich und erlaubte aufmerksamen Beobachtern wertvolle Funde, z. B. von Fossilien. Eine große Bereicherung an Daten brachte aber auch der Bau der Eisenbahnen mit ihren vielen Tunnels und Einschnitten. Der Kreis der Experten war damals noch klein und umfaßte viele Hochschuldozenten oder andere Lehrer, welche somit sehr häufig mit Fragen des Quartärs, aber auch der Praxis konfrontiert wurden. Große, wenn auch stark vereinfachende Ideen wurden entworfen wie etwa das Schema der Eiszeiten oder die Rücksenkung des Alpenkörpers. Es war dies auch die Zeit der großen Streitgespräche, etwa um die Erosionskraft der Gletscher, welche gelegentlich unerwartet aus der Praxis Antwort bekamen - so 1906 beim tragischen Lockergesteinseinbruch unter dem Gasterntal beim Bau des Lötschbergtunnels, als die glaziale Übertiefung handgreiflich bewiesen wurde.

Vor dem Ersten Weltkrieg bis ca. 1960, d. h. bis zur vollen Entfaltung der Hochkonjunktur der Nachkriegszeit, verschob sich zwar der Schwerpunkt der Aktivität der angewandten Geologie stark, kaum aber der Kreis der Experten. Die Erdwissenschaften entfalteten sich rasch, blieben aber doch noch überschaubar. Große Bedeutung gewann nun die Erstellung von Straßen und Kraftwerken, dies bei zunehmendem Einsatz von Maschinen und schwindender Möglichkeit direkter Beobachtungen. Techniken der Bodenerkundung mit verschiedenartigen Sondierungen, mit Geophysik und mit Laboruntersuchungen wurden entwickelt, doch blieb ihre Anwendung vorerst sparsam und lokal. Das Bild des Quartärs erfuhr zahlreiche, wenn auch oft schwer

Conrad Schindler, Prof., Dr., Ingenieurgeologie, ETH-Hönggerberg, $\mathrm{CH}$-8093 Zürich 
interpretierbare Komplikationen (z. B. «Mittelterrassenschotter» oder die komplexen, nur zum Teil publizierten Resultate von P. BECK (1933) im Berner Oberland).

\section{Verhältnisse seit 1960}

Mit dem Aufkommen der Hochkonjunktur setzte eine explosive Ausweitung der Aktivitäten in der angewandten Geologie ein. Zugleich entwickelten sich die Methoden der Baugrunderkundung und der Bautechnik sehr rasch. Wachsende Hektik und massiver Einsatz von Maschinen drängte die Möglichkeit direkter Beobachtungen am Aufschluß weiter zurück.

Nun erst begannen sich Ingenieurgeologie und $\mathrm{Hy}$ drogeologie selbständig und rasch zu entwickeln, dies parallel zu einer zunehmenden Aufsplitterung der Erdwissenschaften. Einerseits ergab sich daraus eine noch heute wachsende Spezialisierung, andererseits entstand in der Schweiz eine zunehmende Zahl beratender geologischer Büros. Diese übernahmen weitgehend die Aufgaben der angewandten Geologie, welche einst von den Hochschulen maßgebend mitgetragen worden war. In unsern Nachbarländern wurden statt dessen Landesämter mit einem Großteil der entsprechenden Aufgaben betraut. An vielen Orten entwickelte sich eine bedauerliche Abkoppelung der geologischen Institute von den Problemen der Praxis - und damit auch von den dort gewonnenen Kenntnissen über unsern Boden. Faszinierender als Lockergesteine und Quartär wirkte für die meisten die Anwendung neuer, immer raffinierter werdender Untersuchungsmethoden oder z. B. die Entwicklung der Plattentektonik.

Während den letzten 25 Jahren wurde eine erstaunliche Zahl von Sondierungen abgetieft - in Zürich allein dürften dies mindestens 10000 sein. In regionalen Grundwasseruntersuchungen wurde der Aufbau der Lockergesteine und der Verlauf der Felsoberfläche erkundet. Der Bau von Autobahnen ließ in unserem Land langgezogene Streifen mit geologischer Detailkartierung entstehen, begleitet von einer Unzahl von Sondierungen aller Art.

Angesichts dieser Fülle von Daten mag erstaunlich wirken, daß gleichzeitig unsere Kenntnisse über das Quartär nur in relativ bescheidenem Maße wuchsen. Eine große Zahl neuer Fakten wurde zwar bekannt. Beim unbefangenen Beobachter konnte im letzten Jahrzehnt aber gelegentlich eher der Eindruck von steigender Komplikation, ja Konfusion als von zunehmender Klarheit entstehen. Um dies zu erklären, möchte ich vorerst an zwei Beispielen kurz zeigen, was für die Lösung ingenieurgeologischer oder hydrogeologischer Probleme wesentlich ist und was nicht.

\section{Beispiel Linthebene bei Reichenburg (Kt. Schwyz)}

Wie auf Abb. 1 zu sehen, stieß bei Reichenburg einst eine aus fluviatil transportierten Kiesen und Sanden bestehende Plattform weit in die Linthebene vor. Es handelt sich dabei um eine am Rande der Ebene durch Schmelzwasser geschüttete Kame-Terrasse. welche in einem kurzen Stillstand während dem Rückzug des Linth-Rheingletschers entstand. Eine klassisch ausgebildete Diskordanz zwischen schiefstehenden Deltaschichten und flachliegenden Übergußschichten beweist, daß der Spiegel des Zürichsees zur Zeit dieser Ablagerung um Kote $417 \mathrm{~m}$ schwankte (heute um $406 \mathrm{~m}$ ). Diese wertvollen Kiesvorkommen wurden nördlich der Bahnlinie vorerst bis auf das Niveau der Ebene abgebaut. In den Jahren 1972/73 folgte massiver Aushub bis zu $20 \mathrm{~m}$ in den Untergrund. Dies war allerdings nur unter Absenkung des Grundwasserspiegels möglich: Laufend wurden ca. $1400 \mathrm{l} / \mathrm{min}$ abgepumpt! Ende 1973 wurde man auf massive Setzungen aufmerksam und stellte fest, daß sich die umgebende Linthebene auf ca. $1 \mathrm{~km}^{2}$ Fläche um bis zu $50 \mathrm{~cm}$ gesenkt hatte, dies aber sehr ungleichmäßig. Es entstanden unliebsame Folgen insbesondere für den kurz zuvor fertiggestellten Autobahnzubringer von Reichenburg zur N3 samt zugehörigen Bauwerken. Glücklicherweise standen im kritischen Bereich zahlreiche Bohrungen, Wasserspiegelmessungen und Fixpunkte zur Verfügung, so da $\beta$ in Kombination mit Zusatzuntersuchungen folgendes Bild ermittelt werden konnte (Abb. 1 und 2):

Unmittelbar nach Ablagerung der Kame-Terrasse überfuhr der Gletscher deren rückwärtigen Teil, was eine Verdichtung des Korngefüges bewirkte (wegen Eisüberlastung; zudem verdichtete der vorstoßende Gletscher aber auch sein Vorfeld). Die Moräne wie auch die kurz nachher in einem lokalen Staubecken seitlich des Eises abgelagerten feinkörnigen Sedimente bildeten eine schlecht durchlässige Decke, weshalb das vom Hang her zufließende Grundwasser nach N und NW geleitet wurde und dann in die Linthebene ausfloß. Die hier anzutreffenden Lockergesteine sind sehr jung und wurden hauptsächlich durch die Linth, aber auch durch Seitenbäche abgelagert. Unser Hügel ragte einst weit in einen tiefen See, dessen Spiegel zur Zeit der Verlandung um 404-407 m geschwankt haben dürfte. Die Ablagerungen unter Seespiegel sind größtenteils sandig bis tonig und enthalten viel organisches Material, seltener Linsen von Kies und Sand. Bei der Verlandung mäandrierten Fluß- und Bachläufe zwischen Sumpf und Überschwemmungsgebieten. Es entstanden bei Grundwasserspiegel nahe der Oberfläche außerordentlich weich gelagerte feinkörnige Schichten mit viel organischen Resten und Lagen von Torf, durch welche sich kiesige bis sandige fluviatile Ablagerungen schlängeln. 


\begin{tabular}{|lll} 
Abb. 1 Randliche Gebiete der Linthebene bei Reichenburg (SZ): Geologische Karte \\
Fig. 1 Geological map of the border of Linth plain near Reichenburg
\end{tabular}

LEGENDE (Abb. 2)

IIIII tonig,organische Resten

2.H. Silt-Feinsand junge Ablagerungen Linthebene

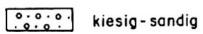

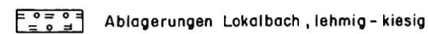

$\because::$ : Kome-Terrasse, über gusschichten, kiesig

$0^{\circ} 0^{\circ}$ Kame - Terrasse, Deltaschichten, kiesig-sandio

$\Delta \triangle$ Moröne und verschürfte Kiese und Sande

$-\_-$eiszeitliche Staubeckenoblagerungen, weich, feinkörnig

$\models \quad$ natürlicher Wosserspiegel (in Piezometer)

|- abgesenkter Wosserspiegel ( in Piezometer)

土.- obgesenkter Grundwosserspiegel ( 1974 )

- +_- Loge ehem. Seespiegel Linthebene

I Kernbohruno

Abb. 2 Geologisches Profil nördlich von Reichenburg

Fig. 2 Geological profile north of Reichenburg

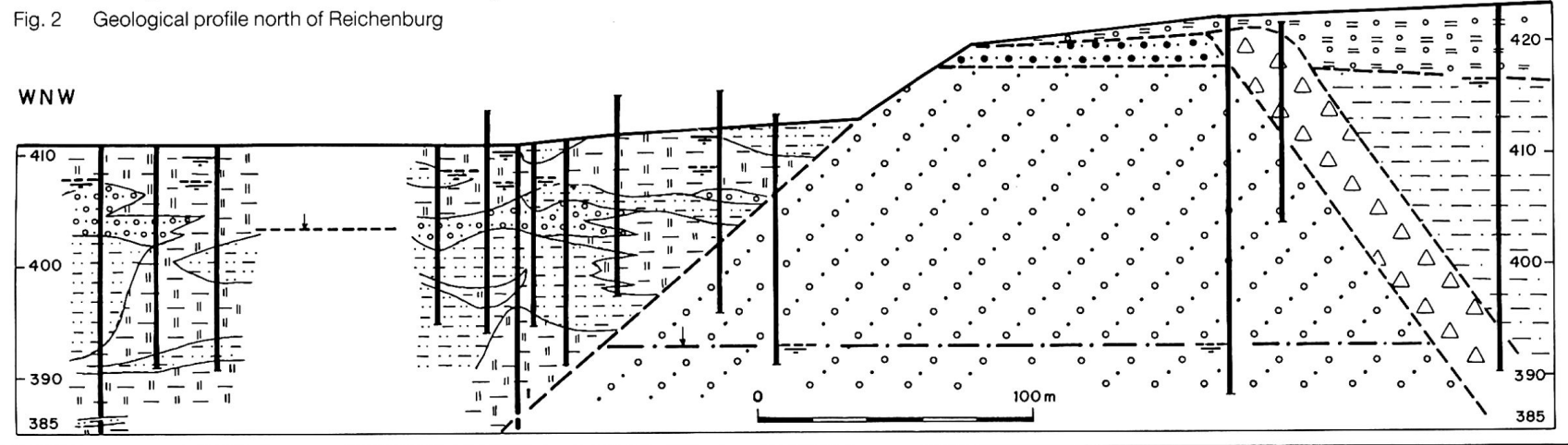


Das Wasser ließ sich in den durchlässigen Sedimenten der Kame-Terrasse mühelos durch Abpumpen absenken. Von den dauernd geförderten $1400 \mathrm{l} / \mathrm{min}$ stammten rund $600 \mathrm{l} / \mathrm{min}$ vom Hang; der Rest entfloß den jungen Sedimenten der Ebene, welche generell wenig durchlässig sind. Die Entwässerung mußte hier deshalb den ehemaligen Bach-und Flußläufen folgen, konnte sich ihnen entlang aber sehr weit ausbreiten. Interessanterweise fiel der Grundwasserspiegel in der Ebene höchstens $2,5 \mathrm{~m}$ unter den ursprünglichen Stand, d. h. bis ca. Kote $407 \mathrm{~m}$. Dies entspricht offenbar der tiefsten Kontaktstelle zwischen Kame-Terrasse und jungen Flußkiesen.

Die Trockenlegung von Lockergestein infolge Spiegelabsenkung erreichte also innerhalb der KameTerrasse sehr großes Ausmaß, bewirkte aber kaum Setzungen. Neben Grobkörnigkeit und Fehlen von Ton und organischen Bestandteilen wirkte sich hier das Auffahren des Gletschers aus (Überkonsolidation). Umgekehrt löste die bescheidene Absenkung innerhalb der jungen Ablagerungen in der Ebene sehr große, weitflächige Setzungen aus. Der Grundwasserspiegel war zuvor nie wesentlich unter die Terrainoberfläche gesunken, so daß die sehr jungen Sedimente wenig konsolidierten. Nun wurden einerseits tonige bis torfige Sedimente entwässert, andererseits entstand durch Wegfall des Auftriebs eine großflächige Mehrbelastung des Untergrunds. Da aber die Drainage sich nicht gleichmäßig auswirken konnte und zudem das Schichtprofil stark wechselt, schwankt auch das Ausmaß der Setzungen massiv und unberechenbar (Abb. 1).

Was zeigt uns dieses Beispiel? Ingenieurgeologie und Hydrogeologie sind sehr stark an scheinbar unwichtigen Details interessiert: Geometrie der KiesSand-Ablagerungen in Kame-Terrasse und Ebene/ Details des Schichtprofils undWechsel von Bohrung zu Bohrung/Geschichte des Grundwasserspiegels/ Frage, ob der Gletscher sein Vorland nochmals leicht überfuhr/Bodenkennwerte und ihr Streuungsfeld usw. Für unsere Probleme unwesentlich ist dagegen das genaue Alter dieser jungen Lockergesteine.

\section{Beispiel «Silthang» bei Gossau (Kt. St. Gallen)}

Hier sollen Probleme in älteren, von Gletscher überfahrenen Lockergesteinen dargestellt werden. Eine ausführlichere, bodenmechanisch orientierte Darstellung dieser Untersuchungen für die Nationalstraße N1 wurde 1976 publiziert (M. GAUTSCHI und C. SCHINDLER).

Zwischen St. Gallen-Breitfeld und Gossau liegt eine mit Niederterrassenschottern bedeckte Ebene, nördlich von ihr schließt der hier besprochene Hang an (Abb. 3). Während dem Zürichstadium des Hochwürm stieß ein Lappen des Rheingletschers von St. Gallen her kommend hierher vor. Andererseits dämmte bei Gossau ein anderer, von Bischofszell her vordringender Arm das Tal ab, weshalb sich kurzfristig ein Stausee im Raum zwischen beiden Eismassen bildete. Da auf Abb. 3 der Gletscher von Osten her vorstie $\beta$, erscheinen hier gegen rechts hin immer jüngere Moränenwälle. Seitlich und vor dem Eis entstand im See eine warvenartig feingeschichtete Anhäufung von Silt mit tonigen oder feinsandigen Lagen. Höher oben werden die Sedimente gröber und gehen in sandigkiesige Schmelzwasserablagerungen über.

Infolge der für das Zürichstadium charakteristischen Pendelbewegungen der Eisfronten wurden diese Lockergesteine im Profil des «Silthanges» (Abb. 4) nachträglich gestaucht, verschleppt, ohne daß diese Komplikationen oberflächlich zu erkennen wären. Hier finden sich Wälle aus Obermoräne, welche in flacheren Hangteilen durch Becken mit Schmelzwasserablagerungen getrennt werden (Abb. 3). Die Bohrungen und künstlichen Aufschlüsse dagegen zeigten, daß über Fels und Grundmoräne eine dachziegelartig verschuppte, heterogene Folge von Lockergesteinen abgelagert wurde. Der südliche Teil dieser Masse ist nachträglich großflächig abgerutscht, was Anlaß zu sehr grossen bautechnischen Schwierigkeiten gab. Was sind die Gründe für die Rutschbewegungen, welche vermutlich kurz nach Abschmelzen der Eiszunge einsetzten, abgeschwächt bis in die Gegenwart anhielten und durch die Erstellung des Strasseneinschnitts partiell aktiviert wurden?

Eine wichtige Ursache ist in der Durchnässung des Rutschhanges zu suchen, fließt ihm doch dauernd 70-100 l/min Wasser zu, was auf ein bedeutendes Speisungsgebiet hinweist. Dies wirkt erstaunlich, ist der «Silthang" doch vom höher gelegenen Hinterland durch eine Depression fast völlig abgeschnitten (Abb. 3), während der Fels als Wasserlieferant hier nicht in Frage kommt. Die Wasserzufuhr muß demnach durch den schmalen Moränengrat bei Neuchlen erfolgen, was durch Quellaustritte und Bohrungen bestätigt werden konnte.

Man muß also annehmen, daß die parallel zum Wall verlaufenden Stränge von durchlässigen Schmelzwasserablagerungen seitlich durch feinkörnige Seeablagerungen und Moräne abgedichtet werden, dies ähnlich wie im linken Teil von Abb. 4. Dort ist zudem zu erkennen, daß nicht ein einheitlicher, sondern eine Vielzahl von Wasserleitern vorliegt, wobei die Wasserspiegel nicht identisch sind. Da das Abrißbord der Rutschung die Schuppenstruktur und damit die Wasserleiter schleifend schief durchschneidet, ergibt sich eine breitflächige Durchwässerung des unterliegenden Hanges.

Die basale Gleitfläche liegt auf große Strecken wenig über der Grundmoräne, vermutlich meist in den tiefsten, besonders tonigen Seeablagerungen. Im 

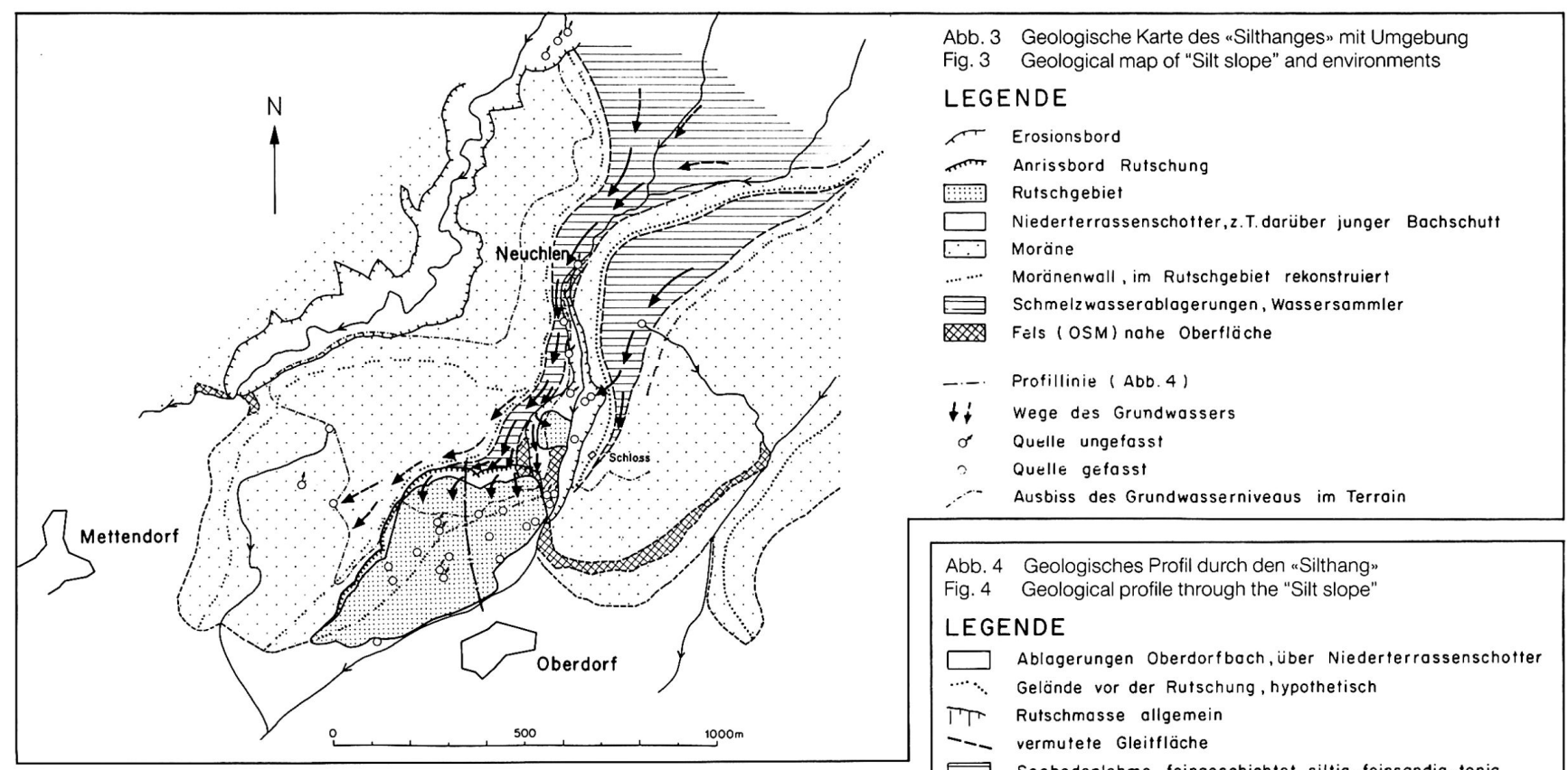

Abb. 4 Geologisches Profil durch den "Silthang"

Fig. 4 Geological profile through the "Silt slope"

\section{LEGENDE}

$\square$ Ablagerungen Oberdorfbach, über Niederterrassenschotter

Gelände vor der Rutschung, hypothetisch

TT Rutschmasse allgemein

- vermutete Gleitfläche

E Seebodenlehme, feingeschichtet, siltig-feinsondig-tonig

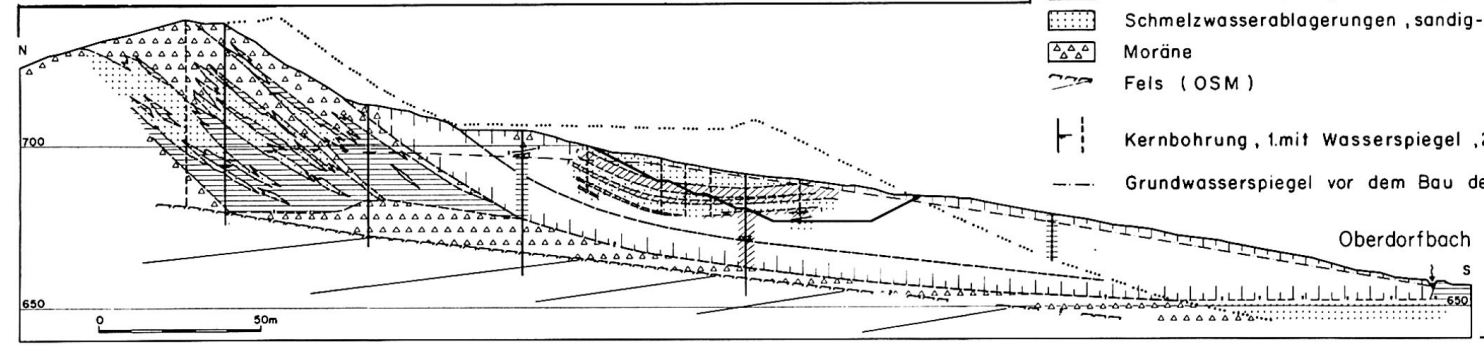


obersten Hangteil folgt sie dagegen dem Gefälle der Schuppengrenzen.

Einige Teilrutschungen oder unstabile künstliche Böschungen gaben Rätsel auf, waren sie doch wenig geneigt und bestanden überwiegend aus Silt oder Feinsand. Die besonders ungünstigen Wasserverhältnisse (evtl. zusätzlich innere Erosion) wirkten zweifellos mit. Ein wesentlicher zusätzlicher Grund ist aber darin zu suchen, daß zufälligerweise lokal die dünnen tonigen Lagen so verliefen wie potentielle Gleitflächen (mittlerer Teil von Abb. 4).

Auch in diesem Beispiel besteht eine enge Wechselbeziehung zwischen Aufbau der Lockergesteine, Hydrogeologie und ingenieurgeologischen Problemen. Vergleichbare Strukturen führen z. B. beim Bau der S-Bahn in Zürich zwischen Kreuzplatz und Hirschengraben zu bedeutenden Schwierigkeiten beim Tunnelbau. Für uns wichtig sind quartärgeologische Details: Pendelbewegungen der Eisstirn/ kurzfristige Bildung von Stauseen/Anisotropie der Ablagerungen in bezug auf Wasserdurchlässigkeit/ zufällige Lage der Schichtgrenzen in abgerutschten Komplexen usw. Die recht bescheidene Überlastung durch Gletschereis bringt eine dramatische Änderung der bodenmechanischen Eigenschaften der ursprünglich sehr weich gelagerten, wenig standfesten Seeablagerungen, denn sie können nun sehr steil angeböscht werden.

\section{Problemkreise zwischen Quartärwissenschaften und Ingenieurgeologie}

\subsection{Datierung}

Bei der Untersuchung des Quartärs wird meist versucht, aus Oberflächenkartierung, z. B. von Moränenwällen, eine regionale zeitliche Abfolge zu rekonstruieren, dies unterstützt von einigen TypusAufschlüssen oder isolierten Bohrungen. Die Pollenanalyse bringt wertvolle zusätzliche Aussagen. Die Extrapolation ihrer Daten wird mit steigendem Alter aber rasch unsicher. Mangels Leitfossilien wäre man auf ununterbrochene Abfolgen von Sedimentation angewiesen. Hat man auf engem Raum viele Sondierungen zurVerfügung, so zeigt sich aber in der Regel, daß in der Schweiz die Ablagerung von Lockergesteinen meist konzentriert während kürzern Perioden erfolgte, so z. B. bei Schaffhausen um das Maximum des Hochwürm (c. SCHINDLER 1985). Zwischen solchen oft sehr heterogenen Schichtstößen liegen schwer erfaßbare Diskordanzen oder Schichtlücken. Absolute Datierungen wären deswegen als Schlüssel von größtem Wert, sind aber vorläufig nur für einen beschränkten letzten Zeitraum zuverlässig. Zudem muß die Möglichkeit von Umlagerungen oder verschiedenartigen andern Störungen berücksichtigt werden.

\subsection{Bodenmechanische Kennwerte}

Wie bereits an Beispielen dargestellt, spielt für die ingenieurgeologische Auswertung der in Feld und Labor gewonnenen Daten die Frage des Alters eine sekundäre Rolle. Dem am Quartär interessierten Geologen wird es deshalb sehr schwerfallen, beim Bauherrn Geld, z. B. für regionale Auswertung, Pollenanalyse oder ${ }^{+4} \mathrm{C}$-Altersbestimmungen, flüssigzumachen.

Umgekehrt wird in den Quartärwissenschaften in der Regel die Aussagekraft der bodenmechanischen Kennwerte der Lockergesteine massiv unterschätzt. Schon eine temporäre Austrocknung der Oberflächenschichten, eine kurzfristige starke Absenkung des Grundwasserspiegels oder eine heute wieder abgetragene Überlastung durch Lockergesteine hinterläßt bleibende Spuren. Viel dramatischer sind die Vorgänge beim Überfahren durch den Gletscher, erfolgt doch die Beanspruchung dabei nicht nur statisch, sondern zusätzlich dynamisch und wirkt sich selbst im Vorfeld aus. Auch sehr wenig durchlässige Komplexe werden nach unseren $\mathrm{Er}$ fahrungen rasch überkonsolidiert, wobei allenfalls temporär Porenwasserüberdruck entsteht. Dies wiederum fördert Verscherung und Verschuppung der Lockergesteine. Weiche Lagerung, z. B. von Seebodenlehmkomplexen, schließt unseres Erachtens eindeutig ein Überfahren durch den Gletscher aus und kann als Indiz für die Altersbestimmung dienen - so z. B. bei der Deutung der tiefen Zürichseebohrung (CH.SCHLÜCHTER 1984). Theoretisch ließe sich aus gezielten Versuchen (Plattendruckversuch, Ödometer) sogar die Größe der einstigen Überlast ermitteln; praktisch stößt dieses Verfahren auf bedeutende Probleme.

\subsection{Anisotropie und Heterogenität der Lockergesteine}

Wie an Beispielen gezeigt, spielen die Anisotropie und Heterogenität der Lockergesteine in der Ingenieurgeologie und der Hydrogeologie eine maßgebende Rolle. Diese Faktoren lassen sich durch Sondierungen und Versuche grob erfassen, während generelle Modelle hier wenig nützen. So kann man zwar von Vorstoß- oder Rückzugschottern sprechen. Für die Strömungsrichtung des Grundwassers, Potentialdifferenzen innerhalb eines scheinbar homogenen Kieskörpers usw. werden aber Details im Schüttungsvorgang wichtig wie $z$. B. das Mäandrieren der Einzelläufe (Untersuchungen Grundwasserdargebot im Testgebiet Emmental, NF-Projekt).

Generell muß für unsere Belange eindringlich vor Verallgemeinerungen gewarnt werden: Die Gletscher des Hochwürms mögen z. B. an vielen Orten wenig erodiert haben - an andern wie z. B. im St. Galler Rheintal (M. EBERLE 1987) oder im Seez- 
Walensee-Zürichseetal war das Gegenteil der Fall. Praktische Folge dieser Tatsache sind z. B. große Probleme bei der Fundation des Viadukts der N3 über das Seeztal bei Flums.

\section{Schlußbemerkungen}

Es wäre anzustreben, die Verbindungen zwischen Quartärwissenschaften und angewandter Geologie enger zu gestalten. Der Weg dazu ist aber mühsam. Die Ingenieurgeologen verfügen zwar über große Mengen von Information, doch können sie wissenschaftliche Auswertungen in der Regel nur während ihrer spärlichen Freizeit vornehmen. Solche Kompilationsarbeiten dürfen auch nicht einfach delegiert werden, da der Bearbeiter das Bohrgut mindestens teilweise persönlich untersucht haben sollte. Eine seriöse Auswertung ist außerordentlich arbeitsintensiv. Die Partner auf der Seite der Hochschulen Pollenanalyse, Altersbestimmungen usw. - sind ihrerseits personell und finanziell unterdotiert, weshalb die meisten interdisziplinären Quartärarbeiten bisher nur dank viel Idealismus zustande kamen. Es ist zu hoffen, daß hier eine Entwicklung zum Besseren stattfinde.

\section{Literaturverzeichnis}

BECK, P. (1933): Über das schweizerische und europäische Pliozän und Pleistozän. In: Eclogae geol. Helv. 26/2, 335-437.

BECK, P. (ohne Datum): Das Quartär. Das Pleistozän. Manuskript, dep. beim Büro der Schweiz. geol. Landesaufnahme.

EBERLE, M. (1987): Zur Lockergesteinsfüllung des St. Galler und Liechtensteiner Rheintales. In: Eclogae geol. Helv. Bd 80/1, 193-206.

GAUTSCHI, M. \& SCHINDLER, C. (1976): Stabilitätsprobleme bei einem großen siltreichen Hanganschnitt. Mitt. Schweiz. Ges. Boden- und Felsmechanik Nr. 81.

SCHINDLER, C. (1985): Geologisch-geotechnische Verhältnisse in Schaffhausen und Umgebung. Beitr. Geologie Schweiz, Geotechn. Komm., kleinere Mitteilungen Nr. 74.

SCHLÜCHTER, CH. (1984): Geotechnical properties of Zürichseebohrung sediments. Quaternary Geology of Lake Zürich: An Interdisciplinary Investigation by deep-lake drilling; edited by K. J. Hsü and K. R. Kelts, in Contributions to Sedimentology, 13. 